\title{
CALLUNA VULGARIS (L.) HULL (ERICACEAE) EN DOS TURBERAS DE SPHAGNUM MAGELLANICUM BRID (SPHAGNACEAE) INTERVENIDAS POR LA EXTRACCIÓN DE TURBA: NUEVA ADICIÓN PARA LA FLORA VASCULAR ADVENTICIA DE LA REGIÓN DE MAGALLANES, CHILE
}

\author{
CALLUNA VULGARIS (L.) HULL (ERICACEAE) IN TWO EXPLOITED PEATLANDS OF \\ SPHAGNUM MAGELLANICUM BRID: NEW RECORD FOR ADVENTITIA VASCULAR FLORA \\ OF MAGALLANES, CHILE
}

Nelson Bahamonde ${ }^{1,2}$, Osvaldo J. Vidal1,2 \& Juan M. Henriquez ${ }^{1}$

Calluna vulgaris (Ericaceae), es un arbusto originario de Europa y Âfrica (Press \& Gibbons, 1993). Es una planta acidófila, de crecimiento lento, enano, siempreverde (Gimingham, 1972), usada con fines ornamentales, para medicina natural y producción de miel de abejas (Persano et al. 2004). Su distribución se encuentra relacionada con ambientes de páramos o de tundra, tolerando un $\mathrm{pH}$ bajo, elementos tóxicos en el suelo (Leake et al. 1989) y escases de nutrientes (Coley et al. 1985).

C. vulgaris crece hasta $150 \mathrm{~cm}$, muy ramificado, con hojas pequeñas de 2,5 a $3,5 \mathrm{~mm}$, opuestas en ángulo agudo, de base obtusa y ápice agudo, las que se encuentran presionadas contra el tallo (Fig. 1 A y C ). Presenta una inflorescencia indefinida en racimo (se estrecha hacia su sección distal a menudo llena de inflorescencias) (Fig. 1A).
Cada flor tiene cuatro pétalos de color rosado y sépalos similares de 3 a $4 \mathrm{~mm}$ con una profunda corola tetra lobulada. Posee entre 6-8 pequeñas brácteas debajo de cada flor, las primeras 4 superiores asemejando a los sépalos (Fig.1B). En Europa florece entre julio y septiembre (Press \& Gibbons, 1993).

C. vulgaris no se encuentra registrada en la base de datos de la Flora del Cono sur del Instituto Darwinion (Zuloaga et al. 2013) y se reporta por primera y única vez en Chile en un catálogo publicado en los Anales de la Universidad de Chile en el siglo XIX (Philippi 1881). Desde entonces no ha sido citada en catálogos de flora vascular de especies introducidas en Chile y no es posible encontrarla en la literatura botánica de la Región de Magallanes (Dollenz, 1983; Domínguez 2010; Domínguez et al. 2006; Espinoza, 1996;

\footnotetext{
${ }^{1}$ Laboratorio de Botánica, Instituto de la Patagonia, Universidad de Magallanes. nebahamo@umag.cl.

${ }^{2}$ Organización no gubernamental de desarrollo medioambiental AMA Torres del Paine, Estancia Cerro Paine S/N, Torres del Payne, Magallanes Chile.
} 


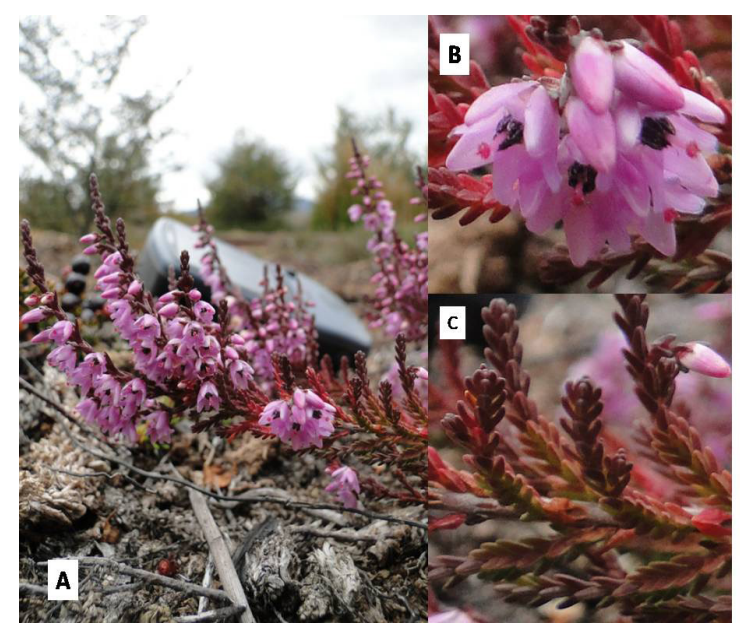

Fig. 1. A) Ejemplar de Calluna vulgaris encontrado en San Juan, B) flor, C) Detalle de las ramificaciones y las hojas.

Henriquez et al. 1995; Matthei, 1995; Marticorena \& Quezada, 1985; Moore \& Godall, 1977; Moore, 1974, 1982; Pisano, 1973; 1977; 1982; 1983; 1994; Phillipi, 1881).

El objetivo de esta nota es informar el hallazgo de C. vulgaris en ambientes generados en turberas abandonadas de la región de Magallanes $e$ indicar antecedentes generales de la especie (origen, hábitat, distribución), su condición actual y las posibles causas de su presencia en este tipo específico de hábitat.

El hallazgo se produjo durante visitas de reconocimiento a 2 turberas en la península de Brunswick, Región de Magallanes. Ambas corresponden a turberas de Sphagnum magellanicum. Una se encuentra actualmente en explotación y se ubica en el sector de San Juan (53³9'36.88”S., 7058'34.12”O.) a $60 \mathrm{~km}$ al sur de Punta Arenas, cubriendo una superficie de $28.000 \mathrm{~m}^{2}$. La segunda turbera (superficie de $2.600 \mathrm{~m}^{2}$ ) se encuentra explotada y abandonada en el sector El Andino, en la periferia de la ciudad de Punta Arenas (53 9'7.48”S., 7057'9.03”O) (Fig. 2).

Muestras de estas plantas fueron herborizadas $y$ depositadas en el herbario del Instituto de la Patagonia (HIP). Como un antecedente, se estimó visualmente la cobertura de las plantas (individuos encontrados) en el sector San

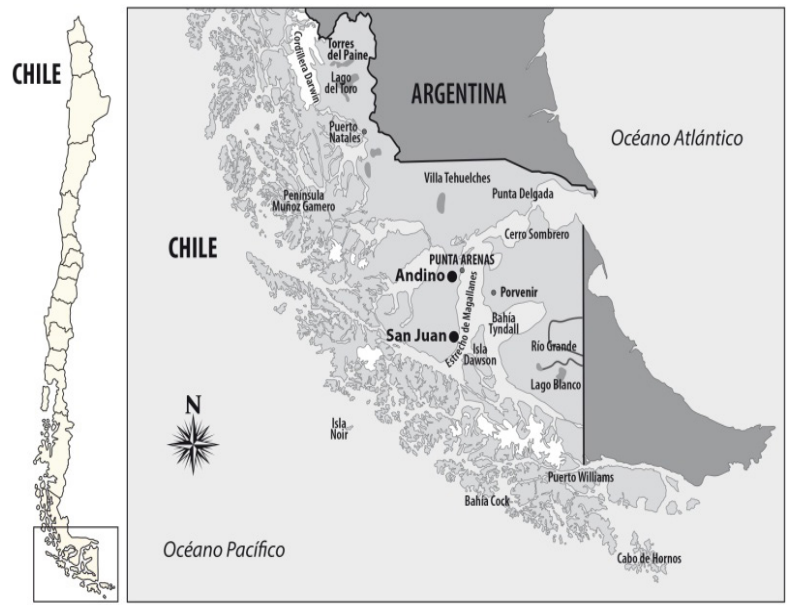

Fig.2. Ubicación geográfica de los sitios de hallazgo de Calluna vulgaris en la región de Magallanes (El Andino y San Juan).

Juan, midiendo ancho máximo y longitud máxima de su follaje, además de la altura de la planta. En El Andino se proyectó un cuadrante de $300 \mathrm{~m}^{2}$ dentro del cual se estimó la cobertura deC. vulgaris y la altura aproximada del matorral.

Para identificar la especie se utilizó una guía de flores de plantas de Gran Bretaña y Europa (Press \& Gibbons 1993). Además fueron revisados los catálogos previos de C. vulgaris (Dollenz, 1983; Domínguez, 2007; 2010; Domínguez et al. 2006, Espinoza, 1996; Henríquez et al. 1995; Matthei, 1995; Marticorena \& Quezada, 1985; Moore \& Godall, 1977; Moore, 1974; 1982; Pisano, 1973; $1977 ; 1982 ; 1983 ; 1994)$ y se realizó un recorrido por establecimientos comerciales que distribuyen plantas para jardín en la ciudad de Punta Arenas.

En la turbera de San Juan se encontraron 2 plantas de C. vulgaris, las que se desarrollan en un sustrato arenoso sobre turba, al costado de una línea de tren, utilizada para el traslado de la turba extraída (Fig. 3 Derecha). Las plantas presentan tallos decumbentes con una altura aproximada de $10 \mathrm{~cm}, y$ una cobertura total de $0,018 \mathrm{~m}^{2}$, lo que corresponde a un $6,4 \times 10^{-5} \%$ del área total de la turbera. En El Andino, se encontró una población en un margen de la turbera, creciendo junto a renovales de Nothofagus antarctica (G. Forst.) Oerst. y matorrales de Berberis microphylla G. Forst. (Fig.3 Izquierda). La población ocupa 


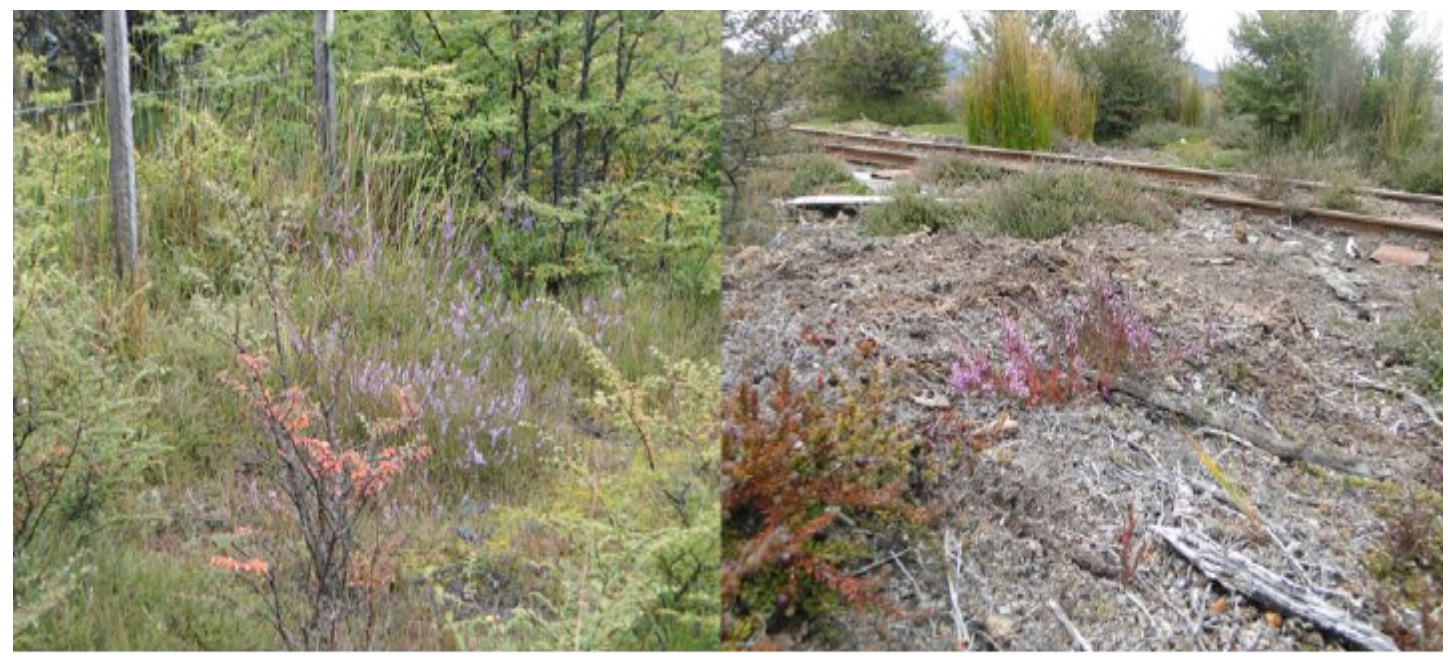

Fig. 3. Izquierda hallazgo turbera Andino y derecha, ejemplares encontrados en la turbera de San Juan.

aproximadamente un $10 \%$ del cuadrante proyectado (aproximadamente $30 \mathrm{~m}^{2}$ ), lo que corresponde a un 1,2\% del área total de la turbera y con una altura promedio de $50 \mathrm{~cm}$.

Luego de revisar catálogos de plantas nativas y exóticas de la región de Magallanes y de Chile, no fue posible encontrar registros de C. vulgaris, excepto en el catálogo de plantas cultivadas en el jardín botánico de Santiago publicado el año 1881 (Phillippi, 1881). Sin embargo, no se reconoce su presencia para Chile, aunque es una planta ornamental muy cultivada en la zona central, bajo el nombre erróneo de "Erica" (Jorge Macaya, com. pers. 2012).

Para la Región de Magallanes no existe un centro de dispersión relacionado con florerías o comercio. La introducción accidental de $C$. vulgaris podría ser el resultado del transporte de diásporas contenidas en la maquinaria de origen europeo utilizada por una empresa que desarrolló actividades extractivas de turba en ambos sitios. La turbera de $\mathrm{El}$ Andino fue la primera explotación realizada por la empresa en 1980 y es en este sector donde se encuentra la mayor cobertura de $C$. vulgaris. En contraposición, la turbera de San Juan, explotada desde el año 2005, presenta solo dos plantas aisladas que se desarrollan en un sustrato de turba cubierto con una mezcla de arena y arcilla glaciar extraída desde el fondo de la turbera, residuos de las faenas de la implementación de los drenajes para la explotación de la turba.

Las modificaciones generales sufridas por ambas turberas (topografía, vegetación, hidrología), favorecen el arribo y establecimiento de especies exóticas como ha sido descrito para otras turberas abandonadas en la región (Dominguez et al., 2012). En este sentido, la presencia de C. vulgaris puede ser considerada como un establecimiento de desarrollo insipiente en San Juan y con características de naturalización para El Andino por la magnitud de su cobertura (Quiroz et al., 2009).

\section{AGADECIMIENTOS}

Se agradece al programa "Bases ambientales, jurídicas y comerciales para el desarrollo sustentable de las turberas de Magallanes" que es financiado por el Gobierno Regional de Magallanes, así como a la SEREMI de Agricultura de la Región de Magallanes y al Instituto de Investigaciones Agropecuarias. También se agradece al Señor Jorge Macaya Berti de la Facultad de Ciencias Agrarias y Forestales de la Universidad de Chile, por suministrar información inédita y a Christian Andrade Sánchez, Biólogo mención Medio 
Ambiente quien participó en los recorridos en la turbera El Andino.

\section{LITERATURA CITADA}

Coley, P.D., Bryant J.P. \& F.S. Chapin (1985). Resource availability and plant antiherbivore defense. Science 230: 895899.

Dollenz, O. (1983). Fitosociología de la reserva forestal "El Parrillar" península de Brunswick, Magallanes. 14: 109-118.

Domínguez, E. (2010). Aportes al conocimiento de la flora y vegetación del Monumento Natural Cueva del Milodón, Región de Magallanes (XII), Chile. Chloris Chilensis Año 13. No 2. URL:http://www. chlorischile.cl

Domínguez, E., Elvebakk, E., Marticorena C. \& A. Pauchard (2006). Plantas introducidas en el Parque Nacional Torres del Paine, Chile. Gayana Botánica 63(2): 131-141.

Dominguez, E. (2007). Catálogo preliminar de gramineas introducidas en la Región de Magallanes (XII), Chile. Chloris Chilensis Año 10. $N^{\circ} 1$. URL:http://www. chlorischile.cl

Dominguez, E., Bahamonde N. \& C. Muñoz (2012). Efectos de la extracción de turba sobre la composición y estructura de una turbera de Sphagnum explotada y abandonada hace 20 años, Chile. Anales Instituto de la Patagonia 40 (2): 37-45.

Epinoza, N. (1996). Malezas presentes en Chile. Instituto de Investigaciones Agropecuarias, C.R.I. Carillanca, Concepción. Editora Aníbal Pinto S.A. 219 p.

Gimingham, C.H. (1972). Ecology of Heathlands. Chapman \& Hall, London, UK. 266 pp

Henriquez, M., Pisano E. \& C. Marticorena (1995). Catálogo de la flora vascular de Magallanes (XII Región), Chile. Anales del Instituto de la Patagonia 23: 6-25.

Leake, J.R., Shaw G. \& D.J. Read (1989). The role of ericoid mycorrhizas in the ecology of ericaceous plants. Agriculture, Ecosystems and the Environment 29: 237- 250.

Matthei, O. (1995). Manual de las malezas que crecen en Chile. Alfabeta Impresores,
Santiago.

Marticorena C. \& M. Quezada (1985). Catálogo de la flora vascular de Chile. Gayana Botánica 42: 5-157.

Moore, D. \& R. Goodall (1977). La flora adventicia de Tierra del Fuego. Anales del Instituto de la Patagonia 8: 263-274.

Moore, D. (1974). Catálogo de las plantas vasculares nativas de Tierra del Fuego. Anales del Instituto de la Patagonia 5: 105-120.

Moore, D. (1983). Flora of Tierra del Fuego. Anthony Nelson England, Missouri Botanical Garden, USA. 396 pp.

Persano, L., Piana L., Bogdanov S., Bentabol A., Gotsiou P., Kerkvliet J., Martin P., Morlot M., Ortiz Valbuena A., Ruoff K. \& Von der Ohe K. (2004). Botanical species giving unifloral honey in Europe. Apidologie 35: 81-93.

Phillippi, R. (1881). Catálogo de las plantas cultivadas para el Jardín Botánico de Santiago hasta el $1^{\circ}$ de mayo de 1881 . Anales Universidad de Chile 59: 519581.

Pisano, E. (1973). Fitogeografía de la península de Brunswick, Magallanes. I Comunidades mesohigromórficas e higromórficas. Anales del Instituto de la Patagonia 4: 141- 206.

Pisano, E. (1977). Fitogeografía de FuegoPatagonia chilena I.- Comunidades vegetales entre las latitudes $52^{\circ}$ y $56^{\circ} \mathrm{S}$. Anales del Instituto de la Patagonia 8: 121- 247.

Pisano, E. (1982). Adiciones a la flora vascular del archipiélago del Cabo de Hornos. Anales del Instituto de la Patagonia 13: 153159.

Pisano, E. (1983). The Magellanic tundra complex. Mires: swamp, bog, fen and moor. Elsevier 10: 295-329.

Pisano, E. (1994). Sectorización Fitogeografía del archipiélago sud patagónico-fueguino. V: Sintaxonimia y distribución de las unidades de vegetación vascular. Anales del Instituto de la Patagonia 21: 6-33.

Press, B. \& B. Gibbons (1993). Wild Flowers of Britain and Europe. New Holland Publishers (UK) Ltd. London. 336 pp.

Quiroz, C., Pauchard, A., Marticorena, A. \& 
L. Cavieres (2009). Manual de plantas invasoras del centro sur de Chile. Laboratorio de Invasiones Biológicas (LIB). Accesadoel 28de Agosto de 2013 en: http:// www.lib.udec.cl/archivos_descargas_pdf/ Manual_de_Plantas_Invasoras_del_CentroSur_de_Chile.pdf
Zuloaga, F., O. Morrone \& M. Belgrano (2011). Catálogo de las Plantas Vasculares del Cono Sur. Versión base de datos en sitio web del Instituto Darwinion, Argentina. URL: http://www.darwin.edu.ar/Proyectos/ FloraArgentina/FA.asp 
